This is a self-archived - parallel published version of this article in the publication archive of the University of Vaasa. It might differ from the original.

\title{
Spatiotemporal Splitting of Distribution Networks into Self-Healing Resilient Microgrids using an Adjustable Interval Optimization
}

Author(s): Gazijahani, Farhad Samadi; Salehi, Javad; Shafie-Khah, Miadreza; Catalao, Joao P. S.

Title: $\quad$ Spatiotemporal Splitting of Distribution Networks into Self-Healing Resilient Microgrids using an Adjustable Interval Optimization

Year: $\quad 2020$

Version: Accepted manuscript

Copyright (C) 2020 IEEE. Personal use of this material is permitted. Permission from IEEE must be obtained for all other uses, in any current or future media, including reprinting/republishing this material for advertising or promotional purposes, creating new collective works, for resale or redistribution to servers or lists, or reuse of any copyrighted component of this work in other works.

Please cite the original version:

Gazijahani, F, S., Salehi, J., Shafie-Khah, M. \& Catalao, J. P. S. (2020). Spatiotemporal Splitting of Distribution Networks into Self-Healing Resilient Microgrids using an Adjustable Interval Optimization. IEEE Transactions on Industrial Informatics (Vol. ahead-of-print No. ahead-of-print). https://doi.org/10.1109/TII.2020.3033560 


\title{
Spatiotemporal Splitting of Distribution Networks into Self-Healing Resilient Microgrids using an Adjustable Interval Optimization
}

\author{
Farhad Samadi Gazijahani, Javad Salehi, Member, IEEE, Miadreza Shafie-khah, Senior Member, IEEE, \\ and João P. S. Catalão, Senior Member, IEEE
}

\begin{abstract}
The distribution networks can convincingly break down into small-scale self-controllable areas, namely microgrids $(\mu G)$, to substitute $\mu \mathrm{Gs}$ arrangements for effectively coping with perturbations. This flexible structure not only could potentially possess the strength to recover quickly, but also ensures the supply of vital loads and preserves functionalities under any contingency. To achieve these targets, this paper examines a novel spatiotemporal algorithm to split the existing network into a set of self-healing $\mu \mathrm{Gs}$. In this endeavor, after designing the $\mu \mathrm{Gs}$ by determining a mix of heterogeneous generation resources and allocating remotely controlled switches, the $\mu$ Gs operational scheduling is decomposed into interconnected and islanded modes. The main intention in the grid-tied state is to maximize the $\mu$ Gs profit while equilibrating load and generation at the islanded state by sectionalizing on-fault area, executing resources rescheduling, network reconfiguration and load shedding when the main grid is interrupted. The proposed problem is formulated as an exact computationally efficient mixed integer linear programming problem relying on the column \& constraint generation framework and an adjustable interval optimization is envisaged to make the $\mu \mathrm{Gs}$ less susceptible against renewables variability. Finally, the effectiveness of the proposed model is adequately assured by performing a realistic case study.
\end{abstract}

Index Terms-Self-healing, Microgrids, Resiliency, Uncertainty, Renewable Energies.

\section{Set and Index}

\section{NOMENCLATURE}

$\begin{array}{ll}b & \text { Index for branches, } b \in n_{b} \\ e & \text { Index for energy storage devices, } e \in N_{E S S} \\ i & \text { Index for DER units, } i \in N_{D E R} \\ l & \text { Index for load point } \\ m & \text { Index for } \mu \mathrm{Gs} \\ n & \text { Index for buses, } n \in n_{n} \\ t & \text { Index for time, } t \in T \\ s w & \text { Index for section switches } \\ t w & \text { Index for tie switches } \\ \boldsymbol{P a r a m e t e r} & \\ I C_{i}^{D E R} & \text { Investment cost of DER [\$/kW] } \\ S_{i}^{D E R} & \text { Capacity of DER units }[\mathrm{kW}] \\ \Lambda_{t, m, n}^{D G} & \text { Operation cost of DG units [\$/kWh] }\end{array}$

F.S. Gazijahani and J. Salehi are with the Department of Electrical Engineering, Azarbaijan Shahid Madani University, Tabriz, Iran (e-mails: f.samadi@azaruniv.ac.ir; j.salehi@azaruniv.ac.ir).

M. Shafie-khah is with School of Technology and Innovation, University of Vaasa, Vaasa, Finland (e-mail: miadreza.shafiekhah@uva.fi).

J.P.S. Catalão is with Faculty of Engineering of the University of Porto and INESC TEC, Porto 4200-465, Portugal (e-mail: catalao@fe.up.pt).
$K_{S W} \quad$ Investment cost of SSW [SUSD]

$K_{t w}$

$K_{e}^{E S S}$

$R U_{d}$

$R D_{d}$

$\bar{D}_{c l, m}$

$\eta_{e}^{C H}$

$\eta_{e}^{D I S}$

$S O C_{e, t}^{i n t}$

$K_{m}^{L S}$

$K_{m}^{R S}$

$G_{b}$

$B_{b}$

Variables

$P_{w}^{W T}$

$P_{v}^{P V}$

$P_{v}^{P V}$
$P_{d}^{D G}$

$P_{d}^{D G}$

$E_{e}^{E S S}$

$\sigma_{w}^{W T}$

$\sigma_{v}^{P V}$

$\sigma_{d}^{D G}$

$u_{t, m, e}^{C H}$

$u_{t, m, e}^{D I S}$

$u_{e, t}^{i d l}$

$\lambda_{t, m}^{S, C}$

$P_{t, m}^{S, C}$

$\lambda_{t, M}^{S, M}$

$P_{t, M}^{S, m}$

$P_{t, m}^{S, M}$

$u_{t, m}^{S, M}$

$\lambda_{t, m}^{B, M}$

$P_{t, M}^{B, M}$

$P_{t, m}^{B, M}$

$u_{t, m}^{B, M}$

$x_{i}$

$\varepsilon_{i}$

$P_{t, m, n}^{D G}$

$\gamma_{t, m, n}$

$P_{t, m, e}^{C H}$

$P_{t, m, e}$

$P_{t, m, e}^{D I S}$

$u_{t, m, e}^{C H}$

$u_{t, m, e}^{D I S}$

$V_{n}, \delta_{n}$

$P_{b}^{\text {flow }}$

$\tau_{s w}$

$\zeta_{t w}$

$q_{d, m}^{u p}$

$q_{d, m}^{d n}$

Investment cost of TSW [\$USD]

Initial state of charge of ESS

Cost of load shedding

Conductance of branches

Susceptance of branches

Generated power of WT $[\mathrm{kW}]$

Generated power of PV $[\mathrm{kW}]$

Energy level of ESS [kWh]

Generated power of DG [kW]

Charging power of ESS

Discharging power of ESS

Power flow at $b$ th branch
Operation cost of ESS units [\$/kWh]

Ramp up limit of dispatchable DG

Ramp down limit of dispatchable DG

Maximum controllable load of each $\mu \mathrm{G}$

Efficiency of ESS at charging mode

Efficiency of ESS at discharging mode

Cost of renewable power spillage

Generated power of $d$ th DG [kW]

Binary variable for operation mode of WT

Binary variable for operation mode of PV

Binary variable for operation mode of DG

Binary variable for charging of ESS

Binary variable for discharging of ESS

Binary variable for idling of ESS devices

Selling price to the consumers at $t$ th hour

Selling power to the consumers at th hour

Selling price to the power market at $t$ th hour

Selling power to the power market at $t$ th hour

Binary variable to indicate the selling status of $\mu \mathrm{G}$

Purchasing price from the power market

Purchasing power from the power market

Binary variable to indicate the buying status of $\mu \mathrm{G}$

Binary variable to determine investment of DER

Integer variable to determine type of DER

Binary variable to show commitment status of DGs

Binary variable to indicate charge mode of ESS

Binary variable to indicate discharge mode of ESS

Magnitude and angle of voltage at $n$th bus

Binary variable for investment decision of SSW

Binary variable for investment decision of TSW

Price for upward power capacity reserve [ $\$ \mathrm{kWh}]$

Price for downward power capacity reserve $[\$ / \mathrm{kWh}]$ 


$\begin{array}{ll}r_{d, m}^{u p} & \text { Upward power capacity reserve provided by DG } \\ r_{d, m}^{d n} & \text { Downward power capacity reserve provided by DG } \\ d_{c l, m} & \text { Controllable load at } \mu \mathrm{G} \\ u_{c l, m} & \text { Binary variable for load commitment state } \\ \kappa_{c l, m} & \text { Per unit value of each load at } \mu \mathrm{G} \\ L S_{t, m} & \text { Load shedding during islanding mode } \\ R S_{t, m} & \text { Renewable power spillage during islanding mode } \\ S D_{T S} & \text { Load interruption in islanding mode of } \mu \mathrm{G} \\ \Delta_{T S} & \text { Duration of islanding mode of } \mu \mathrm{G} \\ N I & \text { Number of islanding in one year } \\ \text { Functions } & \\ I C^{D E R} & \text { Total investment costs of DER units } \\ \text { OC } & \\ f^{\text {island }} & \text { Total operation costs of DER units }\end{array}$

\section{INTRODUCTION}

T $\mathrm{N}$ the last two decades, the renewable energies have rapidly become as an economic and feasible solution in developed countries to counterbalance the quick growth of energy demand and to dramatically lessen the environmental impacts of conventional fossil fuel power plants [1]. However, managing significant levels of heterogeneous distributed energy resources (DERs) along with an extensive and dynamic properties and control points can be cumbersome and overwhelming. On the other hand, the recent significant progress in the control and metering technologies has led to vast efforts to transform conventional electric distribution networks into modern supply-sufficient smallscale zones, so-called microgrids $(\mu \mathrm{G})$, where consumers become prosumers [2].

Actually, the $\mu \mathrm{G}$ would be a low-voltage or medium-voltage distribution system associated with heterogeneous distributed generation (DG) and energy storage systems (ESS) with specified geographic area that can be explicitly regarded as a subset of the power systems to be islanded and still supply in a controlled coordinated fashion, all or part of its customers, thus intrinsically enhancing power system reliability and resiliency during emergency situations. The $\mu \mathrm{G}$ propounds a practical substrate to overcome the challenges subject to integrating renewables with highly volatile nature in power systems through distributed command-and-control strategies. The ability of $\mu \mathrm{Gs}$ to quickly isolate and operate as an independent agent, in case of any disturbance occurred in the upstream network, is the salient trait of smart $\mu \mathrm{Gs}$ in comparison with traditional distribution networks [3].

Besides, one of the most outstanding privileges of $\mu \mathrm{G}$ is its selfrecovery feature that enables it to effectively recover the system to the normal state in the shortest time possible. In other words, it deals with the algorithms for taking preventative and corrective actions to address the faults after they occur, resulting in a reliable and resilient system.

The optimal design, control, and management of $\mu \mathrm{Gs}$ are still challenging task for distribution system operators (DSO) and that is why extensive researches have recently been focused on tackling these crucial issues. The important challenge is to expand an optimized model for efficiently partitioning of distribution systems into a set of self-healing $\mu \mathrm{Gs}$ in a way as to assure both economic and reliability requirements. In fact, the $\mu$ Gs can physically connect to each other at different points by remotely controlled switches (RCS) in order to share the power between themselves (i.e., "spatial") over both normal and emergency conditions (i.e., "temporal") aimed at increasing resiliency and self-healing. The notion of "self-healing" here can be inferred as an intrinsic ability of power system to properly exert corrective and preventative instruments after faults occur to curb the impact of unexpected contingencies on the performance of the system [3].

In the recent years, manifold researches were conducted on the partitioning of distribution systems into the networked $\mu \mathrm{Gs}$ particularly in the event of faults and disturbances [4]-[8]. In particular, Wang et al. [4] proposed a stochastic sectionalization approach for distribution networks to increase the robustness of system against high impact rare events by implementing rollinghorizon optimization approach. A probabilistic model is proposed in [4] for planning of DERs and reactive sources in the form of $\mu \mathrm{G}$ to assure the system reliability criteria. Authors in [6] have considered $\mu \mathrm{Gs}$ as an alternative solution rather than generation and transmission expansion upgrading. In this study, the operational planning of $\mu \mathrm{Gs}$ has been decomposed into a bi-level model with a master objective for $\mu$ Gs planning and a sub-problem for its operational scheduling. In [7], two-stage robust optimization approach is suggested to decide optimal capacity and location of multiple DG units in the $\mu \mathrm{G}$ aimed at minimizing long-term costs and maximizing the profit of $\mu \mathrm{G}$ operator. A $\mu \mathrm{G}$ type selection method has been offered in [8] along with DG planning to determine the combination of hybrid AC/DC $\mu \mathrm{Gs}$ with the aim of minimizing investment and operation costs of DGs, purchasing cost from upstream grid, and cost of reliability reinforcement.

The ESS units can convincingly aid to elevate the proliferation of renewables by providing an additional flexibility for the power system without compromising between affordability, reliability and sustainability. These cutting-edge technologies can broadly bring up considerable benefits for the whole system based on their timescale as power (short duration) or energy (long duration) services. Irrespective of economic and environmental benefits of renewables, the intermittent nature of these resources entails significant challenges in the safe and trustworthy operation of $\mu \mathrm{Gs}$. In this sense, various techniques with different properties are enlarged to tackle the uncertainties pertaining to renewable productions [9].

As a handful the whole sack, scenario-based programming [4], robust optimization [6],[7], and information gap decision theory [10] are extensively employed for dealing with uncertainty. The planning of micro-turbines and WT has been carried out in [11] based on probability-weighted robust optimization, in which the uncertainty induced by WT and consumption has been taken into account. Arefifar et al. [12] proposed a probabilistic self-healing operational planning model to effectively allocate various DGs within the MGs considering unexpected contingencies and reliability indices. An adaptive robust optimization framework has been suggested in [13] to optimally manage the hybrid AC/DC $\mu \mathrm{Gs}$ regarding to the mutual interaction between $\mathrm{AC} / \mathrm{DC} \mu \mathrm{Gs}$, aimed at acquiring a robust decision for whole system. Reference [14] presented a min-max-min model to operate $\mathrm{AC} / \mathrm{DC} \mu \mathrm{Gs}$ in the islanding mode considering the degradation of energy storage. In this work, the startup/shutdown state of DGs is determined at the first stage, while dispatching the DG outputs at the second stage (i.e., economic dispatch). Qiu et al. [15] expanded a multiple-timescale rolling horizon optimization for intraday operation of AC/DC $\mu \mathrm{Gs}$ by means of distributionally robust optimization method.

Depending on the complexity and intentions of the $\mu \mathrm{G}$ planning, it can be modeled through different mathematical foundations such as multi-objective modelling [16], bi-level model [6,25], weighted sum method [17] and two-stage programming [18,19]. For instance, a robust bi-level approach is reported in [20] to cope with uncertain physical and financial information subject to $\mu \mathrm{G}$ planning problem, where investment decision is considered as master 
problem and operation scheduling is also executed as sub-problem. A reconfigurable topology is presented in [21] for the $\mu$ Gs constructions to increase the robustness of distribution system against uncertainty by providing a grid flexibility via automatic switches. This commutable structure provides a superior opportunity to quickly restore the crashed loads due to occurrence of contingency and can substantially enhance the penetration of $\mu \mathrm{Gs}$ in the future distribution systems as well. To address the uncertainties subject to renewables in the hybrid MGs, a multiinterval robust approach has been executed in Ref. [22], in which the impacts of charging rate and the state of charge on the degradation of ESS units are examined by dynamic energy storage degradation model. Moreover, to achieve a fair trade-off between DSO and MG operators, a multi-objective framework is proposed in [23] for optimal operation of grid-connected MGs and solved by a combination process based on self-adaptive genetic algorithm and non-linear programming. In this paper, at first an economic dispatch problem is implemented within the distribution system in order to achieve power loss reduction and voltage profile improvement. Subsequently, the MG operators based on the results of first stage, determine the operational strategy of DGs in MGs.

To sum up, a comprehensive survey of state-of-the-art on $\mu \mathrm{G}$ planning problem reveals that there are still several important deficiencies that need to be properly addressed. For example, they have mainly focused on the interconnected operation mode of $\mu \mathrm{Gs}$ (i.e., normal operation condition), and the islanded operation mode (i.e., self-healing actions) of $\mu \mathrm{Gs}$ has not been extensively investigated in the $\mu \mathrm{G}$ planning problem [6],[8],[11]. Furthermore, the prior uncertainty management approaches have some drawbacks from computational burden [14] and solution accuracy [32], which developing a suitable method seems to be essential to overcome their weaknesses. Besides, new indices should be developed for reliability and resiliency evaluation of $\mu$ Gs during islanding mode which this critical issue has not been studied so far [12],[25]. Taken together with the studies discussed above, this paper makes essential improvements in the contributions and novelties of the previous works such as modeling an efficient and flexible framework for partitioning of distribution networks into resilient self-healing $\mu \mathrm{Gs}$, and considering uncertainties through enforcing appropriate approaches so as to improve both accuracy and implementation time of the problem. With these motivations in mind, this paper provides the following contributions:

* To develop an efficient and flexible framework for splitting large-scale distribution systems into a cluster of supplysufficient resilient $\mu$ Gs in order to facilitate their management at both normal and self-healing operations.

* To beneficially model the simultaneous development of heterogeneous renewable resources and determining the electrical and geographical boundaries of $\mu$ Gs.

* To consider reliability and resiliency measures in the planning process and propose novel indices for assessing the reliability of the $\mu$ Gs during both interconnected and islanded modes.

* To tackle the uncertainty of the problem via an adjustable interval optimization and perform a column \& constraint generation $(\mathrm{C} \& C G)$ approach to solve the problem at hand.

The remainder of this paper is organized as follows: Section II mathematically formulates the proposed operational planning problem; Section III introduces the solution algorithms to appropriately cope with the uncertainty and solve the problem; Section IV represents the simulation cases to demonstrate the benefits and usefulness of the proposed model. Finally, Section V concludes the paper.

\section{PROBLEM FORMULATION}

In spite of promising future of renewable energies, the operators are facing new challenges in maintaining the reliability and security of systems at an admissible and adequate level. The $\mu$ Gs with convenient and intelligent control infrastructure can be considered as one of the ideal solutions to resolve these vital problems. The $\mu \mathrm{Gs}$ can also sell their surplus power to the main grid and in emergency situations such as occurrence of contingencies, the $\mu \mathrm{Gs}$ can separate themselves from the rest of the network and locally supply their own demand independently, i.e., autonomous mode.

The process of converting large-scale distribution systems into networked supply-sufficient $\mu$ Gs includes two basic phases; i) transforming passive distribution system into active one through integration of various DERs and ESS; ii) forming the system as networked $\mu \mathrm{Gs}$ by specification of electrical and geographical boundaries of $\mu$ Gs by allocating RCS. It should be mentioned that the dividing of distribution networks into a set of $\mu$ Gs should be performed considering several important criteria:

- Each $\mu \mathrm{G}$ must have at least one dispatchable DG (as a black start unit for self-recovery)

- Each $\mu \mathrm{G}$ must have enough generation capacity for supplying critical loads (supply-sufficient)

- Each $\mu \mathrm{G}$ must satisfy the required reliability limits at the islanded mode (self-healing)

- Each $\mu \mathrm{G}$ should be able to match the generation and consumption to retain frequency stability

- Each $\mu \mathrm{G}$ should have adequate volt/var control capabilities to maintain voltage profile

\section{A. Mathematical formulation for $\mu G$ planning}

The main intentions for splitting distribution systems into selfhealing $\mu \mathrm{Gs}$ are to maximize the profit of DSO in the normal operation mode and minimize the load shedding in the islanding mode. Indeed, at the normal operation state, the DSO aims to reduce its procurement costs by optimally dispatching its own DERs and exchanging powers among $\mu$ Gs while following a fault occurred on the network the $\mu$ Gs enter islanding mode to preserve the reliability of the system as high as possible. The main objective at the islanded mode is to maximize system reliability rather than earning economic benefits, by employing various self-healing actions like network reconfiguration, DG rescheduling and load shedding. The on-outage area will be sectionalized by RCS and subsequently the available DERs in the unscathed area will be redispatched to supply the critical demand.

1) Objective functions: It had better develop a linear or even convex model for the problem because of their convergence and optimality. The proposed profit maximization problem can be formulated as the difference between the revenue earned and the cost incurred during planning horizon time (1). The generated revenue is due to sales of power to consumers and power market, which can be seen at the first two terms of equation (2), and also incurred costs include economic costs associated with investing and operation of DER and RCS installed on the $\mu \mathrm{Gs}$, as illustrated at the last term of (2). Note that in this work the investment and operation costs of DER $\left(Z^{D E R}\right)$ and $\operatorname{RCS}\left(Z^{S W}\right)$ are converted to cost-based models to be one single objective function (3).

$$
\text { Max Profit }=\text { Revenue }- \text { Cost }
$$




$$
\begin{array}{r}
\text { Revenue }=\sum_{t}\left\{\sum_{m} \lambda_{t, m}^{S, C} P_{t, m}^{S, C}+\sum_{m} \lambda_{t, m}^{S, M} u_{t, m}^{S, M} P_{t, m}^{S, M}\right. \\
\left.-\sum_{m} \lambda_{t, m}^{B, M} u_{t, m}^{B, M} P_{t, m}^{B, M}\right\} \\
\text { Cost }=Z^{D E R}+Z^{S W}
\end{array}
$$

Here, the economic costs subject to DER consists of two main parts, planning expenditure $\left(I C^{D E R}\right)$ and operation cost $\left(O C^{D E R}\right)$ in grid-tied mode of $\mu$ Gs as presented in (4). Equation (5) denotes the investment costs of DER and also equation (6) refers to annual operation costs of $\mu$ Gs during planning horizon time (T).

$$
\begin{gathered}
Z^{D E R}=I C^{D E R}+O C^{D E R} \\
I C^{D E R}=\sum_{i=1}^{N_{D E R}} x_{i} \varepsilon_{i} I C_{i}^{D E R} S_{i}^{D E R}, i \in(D E R) \\
O C^{D E R}=T \times 365 \times \sum_{t}\left\{\sum_{m} \sum_{n} \gamma_{t, m, n} \Lambda_{t, m, n}^{D G} P_{t, m, n}^{D G}\right. \\
\left.+\sum_{m} \sum_{e} K_{e}^{E S S}\left(u_{t, m, e}^{C H} P_{t, m, e}^{C H}+u_{t, m, e}^{D I S} P_{t, m, e}^{D I S}\right)\right\}
\end{gathered}
$$

Equation (7) shows the total investment costs of RCS installed on the system for clustering distribution grid into a set of networked $\mu$ Gs. In the proposed method, two different switches are installed in which section switches (SSW) are installed on the lines of networks in order to sectionalize the system into the self-healing $\mu$ Gs (i.e., spatial partitioning of $\mu \mathrm{Gs}$ ) and tie switches (TSW) that are located between different feeders of system to change the communications of $\mu \mathrm{Gs}$ for increasing their resistance against contingencies (i.e., temporal partitioning of $\mu \mathrm{Gs}$ ). Equation (8) illustrates the capital costs of SSW and TSW mathematically.

$$
\begin{gathered}
Z^{S W}=I C^{S S W}+I C^{T S W} \\
Z^{S W}=\left(\sum_{s w} \tau_{s w} K_{s w}\right)+\left(\sum_{t w} \varsigma_{t w} K_{t w}\right)
\end{gathered}
$$

2) Technical Constraints: The technical constraints mentioned below cover several aspects of the problem consisting limitations on the operational and planning variables such as power mismatch within the $\mu \mathrm{Gs}$ along with the technical constraints pertaining to different DER installed on the $\mu$ Gs.

a) Minimum/maximum capacities of DER: These constraints enforce minimum/maximum installable capacities for DER that scheduled to be install on the $\mu$ Gs (9)-(12). Notably, the total capacity of DERs installed at each $\mu \mathrm{G}$ should be satisfied at least its local load $\left(P_{m}^{\text {load }}\right)$ as expressed in (13) based on required DER penetration index $\left(0 \leq P I^{D E R} \leq 1\right)$.

$$
\begin{aligned}
& \underline{P^{W T}} \sigma_{w}^{W T} \leq P_{w}^{W T} \leq \sigma_{w}^{W T} \overline{P^{W T}}, w \forall N_{W T} \\
& \underline{P^{P V}} \sigma_{v}^{P V} \leq P_{v}^{P V} \leq \sigma_{v}^{P V} \overline{P^{P V}}, v \forall N_{P V} \\
& \underline{P^{D G}} \sigma_{d}^{D G} \leq P_{d}^{D G} \leq \sigma_{d}^{D G} \overline{P^{D G}}, d \forall N_{D G} \\
& \underline{E^{E S S}} u_{e}^{E S S} \leq E_{e}^{E S S} \leq u_{e}^{E S S} \overline{E^{E S S}}, e \forall N_{E S S} \\
& P_{m, w}^{W T}+P_{m, v}^{P V}+P_{m, d}^{D G}+P_{m, e}^{E S S} \leq P I^{D E R} \sum_{l} P_{m, l}^{\text {Load }}, m \forall \mu G
\end{aligned}
$$

b) Maximum installable number of RCS: These constraints restrict the maximum number of SSW (14) and TSW (15) that can be installed on the network which determine the maximum number of $\mu \mathrm{Gs}$ in the distribution grid.

$$
\begin{aligned}
& \sum_{s w} \tau_{s w} \leq N_{\max }^{S S W} \\
& \sum_{t w} \varsigma_{t w} \leq N_{\max }^{T S W}
\end{aligned}
$$

c) Operational limits of ESS units: The limitations on the charging/discharging powers and state of charge (SOC) of ESS units are fulfilled by equations (16)-(18) where (20) guarantees that ESS works at only one operation mode (i.e., charging or discharging or even idle mode). Moreover, the SOC of each ESS at $t$ th instant could be calculated based on its initial charge as well as charged and discharge powers (21).

$$
\begin{gathered}
0 \leq P_{e, t}^{C H} \leq u_{e, t}^{C H} \overline{P_{e}^{C H}}, t \forall T, e \forall N_{E S S} \\
0 \leq P_{e, t}^{D I S} \leq u_{e, t}^{D I S} \overline{P_{e}^{D I S}}, t \forall T, e \forall N_{E S S} \\
\frac{S O C_{e}^{E S S} \leq S O C_{e, t}^{E S S} \leq \overline{S O C_{e}^{E S S}}, t \forall T, e \forall N_{E S S}}{S O C_{e}^{\text {int }}=E_{e}^{0}, e \forall N_{E S S}} \\
u_{e, t}^{C H}+u_{e, t}^{D I S}+u_{e, t}^{i d l} \leq 1, \quad t \forall T, e \forall N_{E S S} \\
S O C_{e, t}^{E S S}=S O C_{e, t-1}^{E S S}+\eta_{e}^{C H} P_{e, t}^{C H} u_{e, t}^{C H}-1 / \eta_{e}^{D I S} P_{e, t}^{D I S} u_{e, t}^{D I S}
\end{gathered}
$$

d) Power mismatch constraint for each $\mu G$ : Constraint (22) enforces that each $\mu \mathrm{G}$ must have at least one dispatchable DG to regulate the frequency of $\mu \mathrm{G}$ during islanding mode (i.e., cranking power). Besides, at each $\mu \mathrm{G}$, the generations of all DER installed on the $\mu \mathrm{G}$ as well as power imported from main grid must be equal with total load consumption $\left(P_{l, t}^{\text {load }}\right)$, power charged in the ESS units and also power exported to the main grid at any time as (23). This constraint ensures the frequency stabilization at each $\mu \mathrm{G}$ during operation time.

$$
\begin{gathered}
\sum_{i=1}^{D G} x_{i} \geq 1, i \in D G \\
P_{w, t}^{W T}+P_{v, t}^{P V}+P_{d, t}^{D G}+u_{m, t}^{B, M} P_{m, t}^{B, M}+u_{e, t}^{D I S} P_{e, t}^{D I S}= \\
\sum_{l} P_{l, t}^{L o a d}+u_{m, t}^{S, M} P_{m, t}^{S, M}+u_{e, t}^{C H} P_{e, t}^{C H}, t \forall T, m \forall \mu G
\end{gathered}
$$

e) Linearized power flow equation: For the sake of convexity, a linearized model has been utilized [24] to compute the power flows of the system as presented in (24),(25). Here, $\omega_{n, n+1}$ denotes the piecewise linear representation of $\cos \left(\delta_{n}-\delta_{n+1}\right)$.

$$
\begin{gathered}
P_{b}^{\text {flow }}=G_{b}\left(V_{n}-V_{n+1}-\omega_{n, n+1}+1\right)-B_{b}\left(\delta_{n}-\delta_{n+1}\right) \\
Q_{b}^{\text {flow }}=G_{b}\left(V_{n}-V_{n+1}-\omega_{n, n+1}+1\right)-B_{b}\left(\delta_{n}-\delta_{n+1}\right)
\end{gathered}
$$

f) Network constraints: The technical constraints related to distribution network must be kept at their permissible range, which magnitude and angle deviations of voltage at nodes are restricted by equations (26),(27) and also constraint (28) guarantees the allowable power flows at branches.

$$
\begin{array}{cc}
\underline{V_{n}} \leq V_{n} \leq \bar{V}_{n} & \forall t, \forall n \\
\delta_{n}^{\min } \leq \delta_{n} \leq \delta_{n}^{\max } & \forall t, \forall n
\end{array}
$$




$$
P_{b}^{\text {flow }} \leq P_{b}^{\max } \quad \forall t, \forall b
$$

g) Radiality and connectivity constraints: Due to simplification of the protection schemes, the structure of distribution system may often be operated radially. For this purpose, in this paper the graph theory has been used to identify radial topology by means of minimum spanning tree concept [25]. To do this, if the number of branches $\left(n_{b}\right)$ would be equal to the number of buses minus number of substations $\left(n_{n}-n_{s}\right)$ as (29), the structure of system will be radial. On the other hand, if the rank of Laplacian matrix $(L)$ would be equal to the difference between number of buses and substations as (30), therefore, the network will be connected. It is worth mentioning that the Laplacian matrix is calculated from incidence matrix $(I)$ of network as $L=I \times I^{T}$.

$$
\begin{gathered}
n_{b}=n_{n}-n_{s} \\
\operatorname{rank}(L)=n_{n}-n_{s}
\end{gathered}
$$

\section{B. Self-healing Strategy}

The most prominent and underlying step toward achieving selfhealing distribution grid is to constitute cutting edge decentralized and independent agents to control the system in the direction of attaining global optimization for the whole system. Given this background, the best way to manage such a complex system is to break down the distribution grid into the small scale energy zones to improve reliability and technical features of system.

The $\mu$ Gs can operate in parallel to the grid or as an island mode by disconnecting from the utility at the point of common coupling (PCC) during events, i.e., faults, frequency or voltage collapses and etc. When there is a fault on the main grid or other $\mu \mathrm{Gs}$, the $\mu \mathrm{Gs}$ are disconnected from the main grid at the PCC and will enter the islanding state (i.e., self-healing mode) working at autonomous state by controlling the RCS to preliminarily partition faulted area for maintaining power supply to critical loads as much as possible.

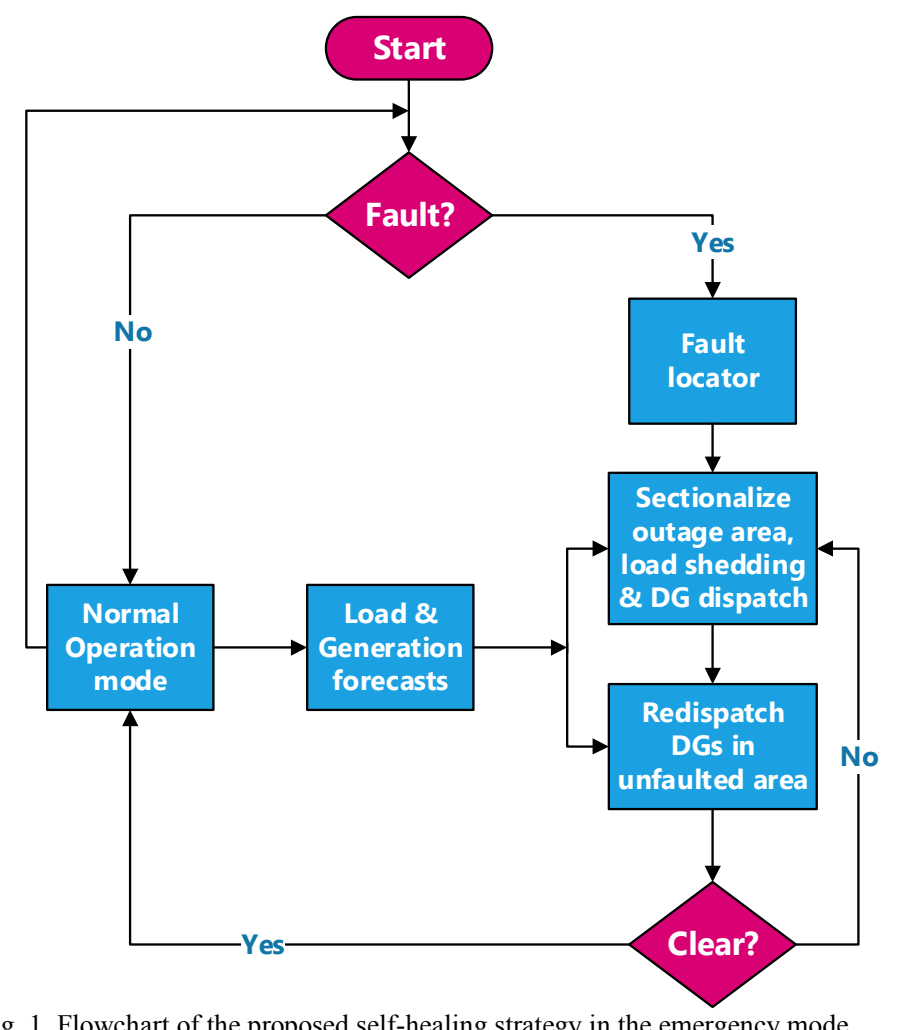

Fig. 1. Flowchart of the proposed self-healing strategy in the emergency mode.
Following a disturbance, the proposed algorithm splits the distribution network into a set of supply-sufficient $\mu$ Gs. The partitioning of distribution networks into a set of $\mu \mathrm{Gs}$ not only speeds up the restoration process, but also reduces the load shedding by sectionalizing the unfaulted areas from faulted one. This is because the combination of DER and RCS (i.e., local generation and grid-forming capabilities) in the form of $\mu \mathrm{Gs}$ can simplify the implementation of local self-healing actions, and therefore accelerate the restoration process by providing additional restoration path and capacity. The main priority in the islanding case is to balance the load-production at each $\mu \mathrm{G}$ by means of coordinately decentralized energy management that satisfy the reliability and security requirements of the system. In the selfhealing mode, the $\mu$ Gs can support other $\mu$ Gs those have power shortage in meeting their critical demand. Indeed, the self-healing is further a power balance problem, instead of optimization one.

The second step is to redispatch the controllable DGs in the unfaulted area since the demand and generation conditions have been changed due to the fault. The system will go back to the normal operation mode after all faults are cleared and continuously check whether there is a fault. Fig. 1 demonstrates the procedures of the proposed comprehensive operation and self-healing strategy.

In the self-healing mode, a quick economic dispatch problem is performed and load dispatch measures are taken to manage the $\mu \mathrm{Gs}$ consumption to avoid from frequency and voltage instability. In other words, the controllable portions of responsible demands can be optimally treated as control variables to stabilize the generationconsumption at each $\mu \mathrm{G}$ and yield the maximum value of the restored loads. On the other hand, the production of controllable DGs must be redispatched to minimize the cost function regarding to technical constraints. The main objective during islanding mode has been represented in (31) which contains the combination of economic and reliability issues as well as technical measures. This objective function must be minimized associated with (32)-(40) and reliability constraints (41),(42).

$$
\begin{aligned}
& f^{i s l a n d}=\sum_{m}\left\{\begin{array}{l}
\sum_{i} C_{i, m}\left(P_{i, m}^{D E R}\right)+\sum_{d}\left(q_{d, m}^{u p} r_{d, m}^{u p}+q_{d, m}^{d n} r_{d, m}^{d n}\right) \\
+\sum_{c l} d_{c l, m} u_{c l, m} \kappa_{c l, m}
\end{array}\right\} \\
& \underline{P_{i, m}^{D E R}} \leq P_{i, m}^{D E R} \leq \overline{P_{i, m, t}^{D E R}}, \quad \forall i, \forall m, \forall t \\
& P_{d, t}^{D G}+r_{d, t}^{u p} \leq u_{d, t} \overline{P_{d}^{D G}}, \quad \forall d, \forall t \\
& P_{d, t}^{D G}-r_{d, t}^{d n} \leq u_{d, t} P_{d}^{D G}, \quad \forall d, \forall t \\
& P_{d, t}^{D G}-P_{d, t-1}^{D G} \leq R U_{d}, \quad \forall d, \forall t \\
& P_{d, t-1}^{D G}-P_{d}^{D G} \leq R D_{d}, \quad \forall d, \forall t \\
& 0 \leq r_{d, t}^{u p} \leq u_{d, t} \overline{r_{d}^{u p}}, \quad \forall d, \forall t \\
& 0 \leq r_{d, t}^{d n} \leq u_{d, t} \overline{r_{d}^{d n}}, \quad \forall d, \forall t \\
& 0 \leq d_{c l, m} \leq \overline{D_{c l, m}}, \quad \forall m, \forall t \\
& \sum_{m}\left\{\sum_{i} P_{i, m}^{D E R}-\sum_{l} P_{l, m}^{\text {load }}+\sum_{c l} d_{c l, m}\right\}=0
\end{aligned}
$$

The main objective in the islanding mode has three different parts as presented in (31); the former is the operating cost of DER that should be minimized, the latter represents the reserve capacity of $\mu \mathrm{Gs}$ and eventually the last part declares the load shedding during 
islanding mode of $\mu \mathrm{Gs}$. The constraints during islanding mode of $\mu \mathrm{Gs}$ consist prohibited operation zones of DER available in the $\mu$ Gs (32), DG generation plus reserve limits (33),(34), DG ramping up/down limits (35),(36), DG upward/downward scheduled reserves limits (37),(38), allowable value of controllable loads (39) and power balance constraint (40).

In spite of the fact that various reliability indices have already defined in the IEEE Standard 1366-2012 [26] to calculate reliability level of distribution networks at interconnected mode, they are not capable to appropriately represent the system behavior at the islanding mode. To this end, new indices are needed to describe the distinct characteristics brought by $\mu \mathrm{Gs}$ in the emergency state. In this paper, two new metrics are employed to evaluate the reliability of $\mu \mathrm{Gs}$ at islanding mode [27]. The new metrics proposed in this paper include operation reliability indices in islanded mode and customer-based reliability indices, which will complement existing reliability indices by measuring specific aspects. In the islanding mode, the main objective is to maintain the power balance while satisfying both reliability measures:

$$
\begin{gathered}
I E E I=\frac{\sum_{T S} S D_{T S} \cdot \Delta_{T S}}{N I} \leq I E E I^{\text {Target }} \\
I E E D=\frac{\sum_{T S}\left(D_{T S}-P_{T S}\right) \Delta_{T S}}{N I} \leq I E E D^{\text {Target }}
\end{gathered}
$$

Island expected energy interrupted (IEEI) denotes the expected load energy interrupted during islanded mode of a $\mu \mathrm{G}$ due to available power deficiency (41) and also, island expected energy deficiency (IEED) refers to average energy deficiency during islanded mode due to hours when island load exceeds total available island power generation (42).

\section{SOlution Methodology}

\section{A. Adjustable Interval Optimization}

Existing of several major uncertainties such as intermittent productions of renewables make substantial obstacles to optimally use of renewables. Hence, to peruse the effects of these uncertain parameters on the problem, in this paper an interval optimization has been utilized relies on $\mathrm{C} \& \mathrm{CG}$ framework. In the interval optimization, the uncertain parameter is assumed to vary between two minimum and maximum values (i.e., feasible interval) aimed at finding the lower and upper bounds of the outputs at an acceptable computational time [28]. In other words, interval optimization can provide rigorous enclosures of solutions - with absolute certaintyto model objectives by permitting us to compute interval enclosures for the exact values of integrals based on branch-and-bound approaches [29]. Unlike stochastic scenario-based programming that needs to know the PDF of uncertain parameters and imposes considerable computational complexity on the problem, in the interval optimization, we do not entail to possess the PDF of parameters in the decision-making process.

To apply the interval optimization approach on the problem, first, the presented deterministic $\mu$ Gs planning model with relevant inequality and equality constraints are symbolically shown on the basis of their standard form as equations (43)-(45):

$$
\begin{gathered}
\underset{D V}{\operatorname{Max}} F=\operatorname{Profit}[\Psi, \xi] \\
\mathbf{X}(M, N, \Psi) \leq 0 \\
\mathbf{Y}(M, N, \Psi)=0
\end{gathered}
$$

Assuming $\xi$ as uncertain parameter with lower and upper bounds $\xi$, $\bar{\xi}$, the minimum and maximum values can be calculated for objective function instead of expected value by means of equations (46),(47), respectively.

$$
\begin{aligned}
& F^{-}(\Psi)=\min _{\xi} F(\Psi) \\
& F^{+}(\Psi)=\max _{\xi} F(\Psi)
\end{aligned}
$$

After this arithmetic transformation, the interval objective function will be replaced instead of initial one to optimize its bounds regarding given input intervals. In other words, the interval optimization tries to minimize the interval of objective function rather than the worst possible scenario. Toward this direction, both average objective $\left(F^{a v g}(\Psi)\right)$ and also its deviations $\left(F^{d i v}(\Psi)\right)$ should simultaneously be optimized as presented in (48) until the $\mu \mathrm{Gs}$ would be more robust against the uncertainty. Equations (49),(50) show the average and deviation of objective function.

$$
\begin{gathered}
F(\Psi)=\operatorname{Max}\left(F^{a v g}(\Psi) \mid \operatorname{Min}^{d i v}(\Psi)\right) \\
F^{a v g}(\Psi)=\frac{F^{+}(\Psi)+F^{-}(\Psi)}{2} \\
F^{\text {div }}(\Psi)=\frac{F^{+}(\Psi)-F^{-}(\Psi)}{2}
\end{gathered}
$$

It should be stated that one of the important concerns in conjunction with interval optimization is that the model can be very conservative by increasing deviations from the estimated values of uncertain parameters. For this purpose, in this paper an adjustable interval model has been extended rather than conventional one to obviate this worriment. In this approach, an additional penalty factor is added into the islanding objective function (31) that represents the costs related to renewables spillages. In other words, depending on the shortage or surplus of renewable productions compared to forecasted values, respectively, load shedding or renewable spillage (i.e., wind or solar curtailment) can be occurred to balance load and generation at each $\mu \mathrm{G}$. This equation should be minimized subject to constraints (32)-(40).

$$
f^{i s l a n d}=\sum_{m}\left\{\begin{array}{l}
\sum_{i} C_{i, m}\left(P_{i, m}^{D E R}\right)+\sum_{d}\left(q_{d, m}^{u p} r_{d, m}^{u p}+q_{d, m}^{d n} r_{d, m}^{d n}\right)+ \\
\sum_{c l} d_{c l, m} u_{c l, m} \kappa_{c l, m}+\left(K_{m}^{L S} L S_{t, m}+K_{m}^{R S} R S_{t, m}\right)
\end{array}\right\}
$$

\section{B. Proposed $C \& C G$ algorithm}

It goes without saying that the proposed model, which is a minmax-min problem, cannot explicitly be solved using off-the-shelf solvers. To solve this complicated problem, we proposed C\&CG framework to decompose the problem into a sets of solvable problems, i.e., one master problem and some slave problems, and solve it by any off-the-shelf mixed integer linear programming (MILP) solvers [30]. By applying the proposed C\&CG framework on the original problem, the master problem can be formulated as:

$$
\begin{gathered}
\min _{f \in F} A_{0}^{T} f+\xi \\
\text { s.t. } \xi \geq B_{0}^{T} u+C_{0}^{T} z \\
A_{1}^{T} f+B_{1}^{T} u+C_{1}^{T} z=q_{1} \\
A_{2}^{T} f+B_{2}^{T} u+C_{2}^{T} z \leq q_{2}
\end{gathered}
$$


It is obvious that the master problem is a relaxation of the original problem. Thus, it yields a lower bound for main problem (1)-(8). Denote $\hat{f}$ is a solution for the master problem. In addition, the subproblem can be defined as:

$$
\begin{gathered}
\max _{u \in U} \min _{z \in Z} B_{0}^{T} u+C_{0}^{T} z \\
\text { s.t. } A_{1}^{T} \hat{f}+B_{1}^{T} u+C_{1}^{T} z=q_{1} \\
A_{2}^{T} \hat{f}+B_{2}^{T} u+C_{2}^{T} z \leq q_{2}
\end{gathered}
$$

For any given solution $\hat{f}$ that is not optimal, the objective value of the sub-problem would be larger than the true optimal solution of original one. Therefore, the sub-problem yields an upper bound for problem. However, the sub-problem is still a bi-level problem which is difficult to solve. We can reformulate the constraints (56)(58) into complementary constraints using the Karush-KuhnTucker (KKT) optimality conditions as (59)-(61):

$$
\begin{gathered}
A_{1}^{T} \hat{f}+B_{1}^{T} u+C_{1}^{T} z=q_{1} \\
C_{0}^{T}+\varphi^{T} C_{1}+\lambda^{T} C_{2}=0 \\
0 \leq q_{2}-C_{2} z-A_{2} \hat{f}-B_{2} u \perp \lambda \geq 0
\end{gathered}
$$

Where $\varphi$ and $\lambda$ are dual variables of the problem defined in (56)(58). For the sake of convexity, the KKT conditions (61) can be transformed to linear form by applying Big-M method (62),(63):

$$
\begin{gathered}
0 \leq q_{2}-C_{2} z-A_{2} \hat{f}-B_{2} u \leq M . \theta \\
0 \leq \lambda \leq M .(1-\theta)
\end{gathered}
$$

Where $M$ is a large value and $\theta$ is a binary variable. Note that a too large value for $M$ cannot keep the constraint, and also too small value will result to increase the simulation time. So, an iterative based method has been exercised to ascertain the best value of $M$ parameter. In addition, the non-linearities in the objective functions have been linearized by strong duality theory. As a result, by applying linearization methods the constraints of the sub-problem will become as an MILP model. In the $k$ th iteration of C\&CG algorithm, we add new unknown variables $z^{k}$, known variables $\hat{u}^{k-1}$ and corresponding constraints defined as (53)-(55) into the master problem. The basic idea of C\&CG is to create a set of new variables and corresponding constraints and add them to the master problem to refine the first stage decisions. In short, the C\&CG algorithm will decompose the original problem into one master problem (i.e., investing problem) and two subproblem (i.e., one for interconnected operation and another for islanded operation).

The comprehensive procedure of C\&CG can be summarized as follows (In the $k$ th iteration, denote the master problem as $M^{k}$ and the sub-problem as $S^{k}$ ):

- $\quad$ Step 0: Initialize the lower and upper bounds by setting up $L B=-\infty$, and $U B=+\infty$, with the tolerance $\varepsilon \geq 0$ as well as $\hat{u}^{0} \in U, k=1$.

- Step 1: Optimize the master problem $M^{k}\left(\hat{u}^{k-1}\right)$ and then update the lower bound $L B=\max \left\{L B, A_{0}^{T} \hat{f}^{k}+\hat{\xi}^{k}\right\}$ considering the optimal solution as $\left(\hat{f}^{k}, \hat{\xi}^{k}\right)$.

- Step 2: Optimize the subproblem $S^{k}\left(\hat{f}^{k}\right)$ and update the upper bound $U B=\min \left\{U B, A_{0}^{T} \hat{f}^{k}+B_{0}^{T} \hat{u}^{k}+C_{0}^{T} \hat{z}^{k}\right\}$ regarding to the optimal solution $\left(\hat{u}^{k}, \hat{z}^{k}\right)$; update the mathematical form of master problem by enforcing new variables and corresponding constraints, i.e., optimality and feasibility cuts.
- Step 3: With respect to the optimality gap of the problem Gap $=((U B-L B) / L B) \times 100 \%$, if Gap $\leq \varsigma$, optimization process is finished and optimal variables $\hat{f}^{k}$ are reached. Otherwise, set $k=k+1$ and go to Step 1 and repeat the process until the convergence condition is established.

\section{NUMERICAL RESULTS}

In this section, the standard IEEE 33-bus distribution network is employed to apply the proposed approach. The proposed problem is implemented under GAMS environment and subsequently solved by CPLEX 12.5.1 [31]. The technical parameters and levelized cost of energy related to DER units have been borrowed from [32]. It should be stated that in this paper the planning horizon time is assumed to be 5 year (i.e., $T=5$ ), whereas for the sake of simplicity it is assumed that load profiles of $\mu \mathrm{Gs}$ do not change over the year as presented in Fig. 2. Further, the hourly electricity price at retail market is shown in Fig. 3 which obtained from Ontario power market on Sunday 15 April 2018 [33].

The DER units are known as backbone of $\mu \mathrm{Gs}$, which are responsible to supply the demand of $\mu \mathrm{G}$ locally. Therefore, these resources should have enough capacity to meet critical loads of $\mu \mathrm{Gs}$ during islanding mode. The results obtained for DER planning are declared in Table I that shows the optimal location and capacity of DER. Due to technical issues in the production of these resources, their available sizes are provided in the $25 \mathrm{~kW}$ steps. It should be pointed out that the proposed problem is a static one, therefore, all investments will be accomplished at the first year. In addition, the final topology for networked $\mu \mathrm{Gs}$ is graphically depicted in Fig. 4. As can be seen, each $\mu \mathrm{G}$ is encompassed by RCS in the direction of minimizing load curtailment during cascading outages. It is important to note that the total capacity of DER installed at each $\mu \mathrm{G}$ should be greater than its critical loads (i.e., supply-sufficient). Moreover, each $\mu \mathrm{G}$ must have at least one dispatchable resource to control the frequency and voltage of $\mu \mathrm{G}$ in the allowed ranges at the islanding state by responding locally using the droop control approaches, i.e., primary control.

Fig. 5 illustrates the daily power generation of DER units at all constructed $\mu \mathrm{Gs}$ in normal mode by running an economic dispatch at each $\mu \mathrm{G}$. The $\mu \mathrm{Gs}$ are connected to the main grid and imports/exports power from/to the other $\mu$ Gs or main grid aimed at finding an equilibrium pattern to increase its own profit. With respect to this figure, it is obvious that the ESS units play the role of energy broker by charging at low load hours and then discharging at the peak hours. Moreover, dispatchable resources have the duty of supplying the other portion of $\mu$ Gs consumption and eventually the shortage of power, if any, should be purchased from the electricity market.

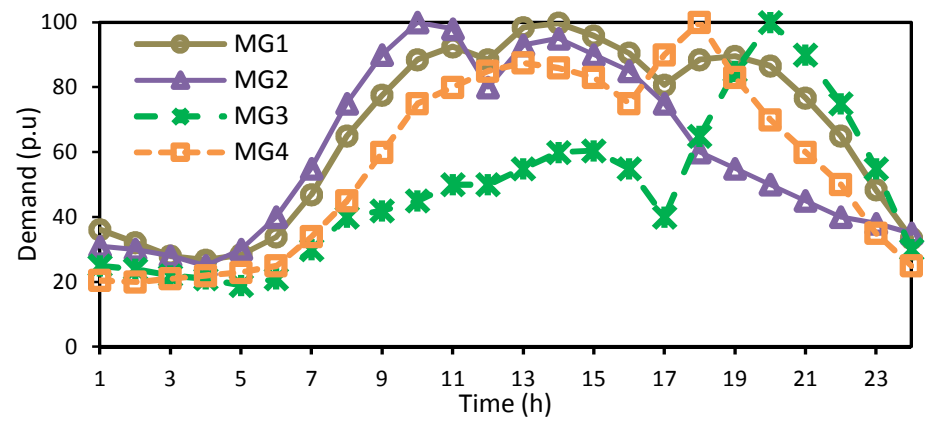

Fig. 2. Consumption patterns of $\mu \mathrm{Gs}$ loads. 


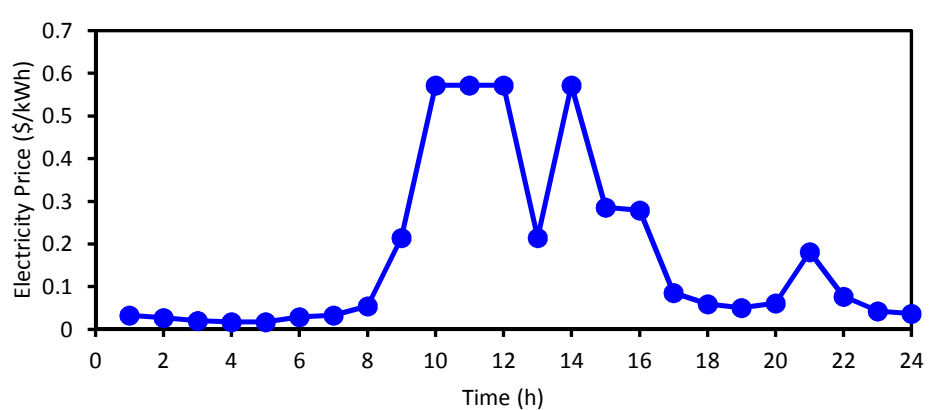

Fig. 3. Electricity price profile at electricity pool.

TABLE I

OPTIMAL PLANNING OF DIFFERENT DER UNITS IN THE NETWORK

\begin{tabular}{ccc} 
DER & Site (bus) & Size $(\mathrm{kW})$ \\
\hline WT & $9,20,24$ & $150,225,350$ \\
PV & $7,17,28$ & $200,200,200$ \\
ESS & $2,15,30$ & $250.200,200$ \\
DDG & $5,11,26,33$ & $300,300,400,300$ \\
\hline
\end{tabular}

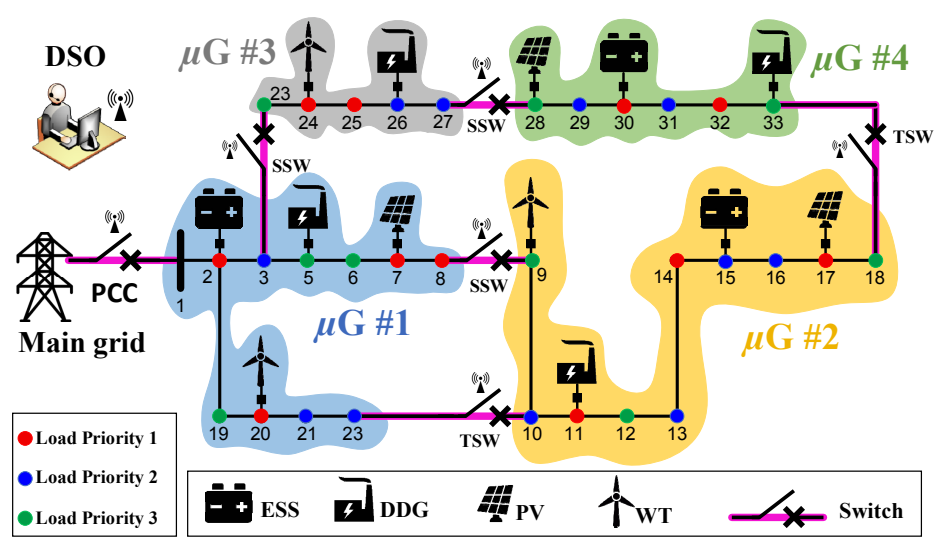

Fig. 4. Final configuration for networked self-healing $\mu$ Gs.

One of the most beneficial features of networked $\mu$ Gs is their ability to transfer the power between the $\mu$ Gs with different users, i.e., residential, commercial, industrial and so on. This issue make the $\mu \mathrm{Gs}$ less susceptible and more robust in conjunction with renewable fluctuations. In other words, the $\mu \mathrm{Gs}$ can supply their peak demand from those $\mu$ Gs that are located in their off-peak hours, e.g. power transaction between a residential $\mu \mathrm{G}$ and commercial one. This power swapping not only minimizes the procurement expenditures of $\mu \mathrm{Gs}$, but also increases the robustness and security of the system against any cascading outage. Fig. 6 declares the power exchanges among $\mu$ Gs for a typical day which the positive values refer to the power sold and the negative values denote the power purchased between a couple of $\mu \mathrm{Gs}$. As can be seen, for instance, $\mu \mathrm{G} \# 1$ sells its extra power from hours 1 up to 19 to the $\mu \mathrm{G} \# 3$ and purchases its power required during hours 20 until 23 from it. That is why, the consumption of $\mu \mathrm{G} \# 3$ over hours 1 to 19 is higher than consumption of $\mu \mathrm{G} \# 1$ and therefore, $\mu \mathrm{G} \# 1$ acts as a backup resource for $\mu \mathrm{G} \# 3$ to supply its demand. In contrary, during hours 20 to 23 , the position of these two $\mu$ Gs are changed and $\mu \mathrm{G} \# 3$ supply the power shortage of $\mu \mathrm{G} \# 1$.

When a fault occurs on a $\mu \mathrm{G}$, the switches installed around the $\mu \mathrm{G}$ detect the fault (by fault and islanding detection algorithms) and separate the deteriorated $\mu \mathrm{G}$ from the rest of intact $\mu \mathrm{Gs}$. After that, the intact $\mu$ Gs are pushed to the islanding mode to supply their own loads independently. Note that in the islanding mode, the priority is with critical loads and unimportant and nonsensitive loads can be cut off, i.e., load shedding, in order to prevent the system from global blackout.

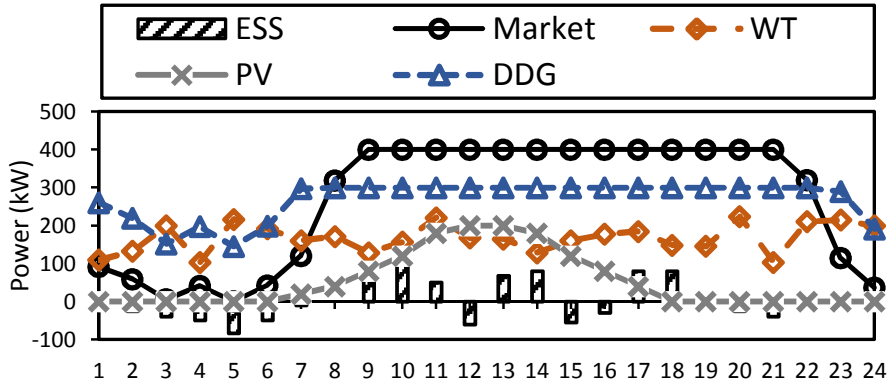

(a) $\mu \mathrm{G} \# 1$

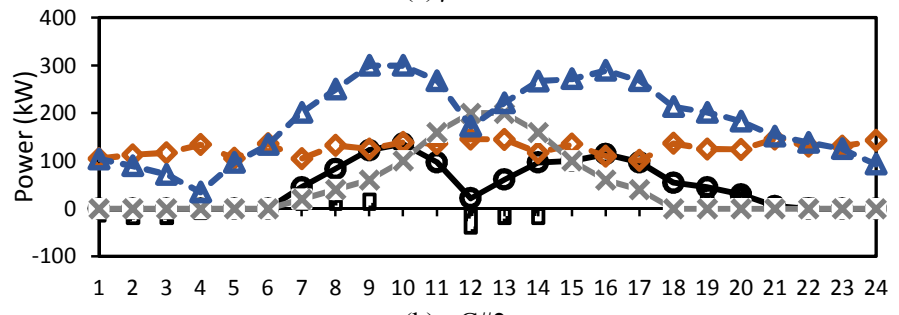

(b) $\mu \mathrm{G} \# 2$

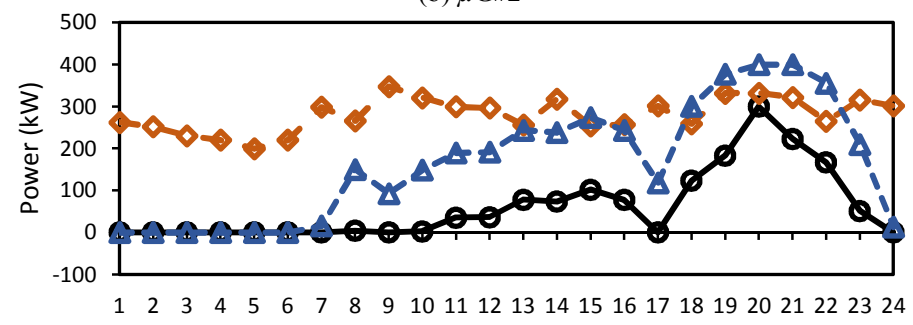

(c) $\mu \mathrm{G} \# 3$

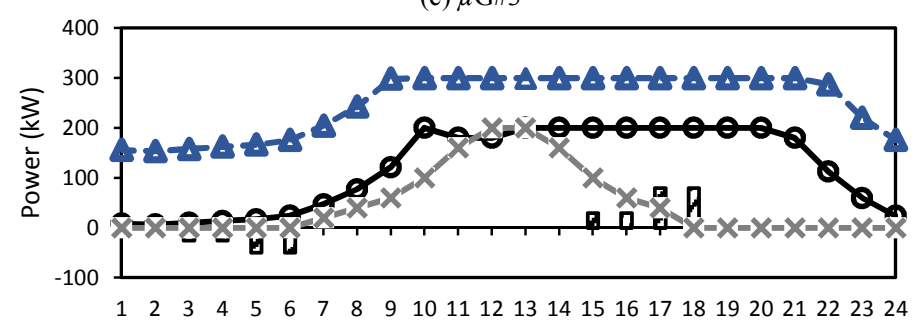

(d) $\mu \mathrm{G} \# 4$

Fig. 5. Daily economic dispatch of $\mu \mathrm{Gs}$ at the interconnected mode.

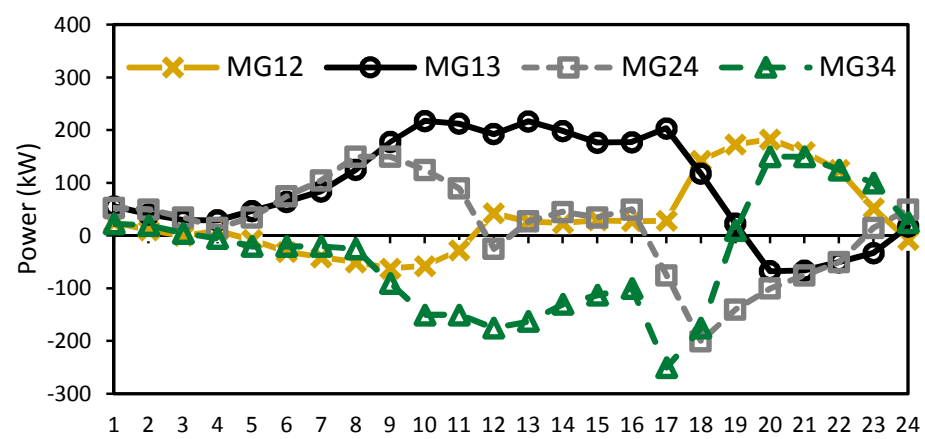

Fig. 6. Power exchange between $\mu$ Gs at the interconnected mode.

Under this situation, the dispatchable DGs have the role to control the frequency of system, i.e., load-generation balancing, and could be considered as a slack bus for islanded $\mu$ Gs. To generally appraise the results gained from the proposed interval approach with deterministic one, a comparative study has been conducted in Table II. With respect to this table, it can be deduced that the proposed approach relatively increases investment and operating costs of system in contradistinction to deterministic state. This is due to the fact that the proposed interval method attempts to 
simultaneously reduce the objective function (cost function) and its fluctuations. Needless to say that the cause of this increase in the economic cost is the swings of renewable generations from their predicted values. On the other hand, it is obvious that the proposed method brings better and more reliable results proportion to deterministic one. In the interval approach, the expected value of load shedding during islanding mode of $\mu \mathrm{Gs}$ has been considerable decreased from $516.12 \mathrm{~kW}$ to $375.2 \mathrm{~kW}$. Similarly, the renewable spillage in the proposed approach has been reduced from 202.68 $\mathrm{kW}$ to $148.06 \mathrm{~kW}$. In the same way, the significant improvements are intuitively observed in the reliability indices of $\mu \mathrm{Gs}$. As ultimate summing up, the proposed interval approach leads to increase the economic expenditures of system by about $5.17 \%$ in exchange of reducing the risk of system. In other words, the value of security against the uncertainties of renewable resources is 44282.57 \$US.

TABLE II

THE IMPACT OF UNCERTAINTY ON THE PROBLEM

\begin{tabular}{ccc}
\hline Objectives & Interval & Deterministic \\
\hline Purchased power (kW) & 11543.66 & 10691.35 \\
Sold power (kW) & 8943.11 & 9278.24 \\
Revenue (\$US) & 122668.21 & 125375.01 \\
Investment cost (\$US) & 586245.25 & 562350.75 \\
Operation cost (\$US) & 318446.63 & 298149.37 \\
Load shedding (kW) & 375.20 & 516.12 \\
Renewable spillage (kW) & 148.06 & 202.68 \\
IEEI (kWh) & 534.77 & 689.41 \\
IEED (kWh) & 351.29 & 437.52 \\
\hline
\end{tabular}

TABLE III

COMPARATIVE STUDY OF METHODS FOR $\mu \mathrm{G}$ PLANNING

\begin{tabular}{cccccc}
\hline Ref. & $\begin{array}{c}\text { Case } \\
\text { study }\end{array}$ & Optimization algorithm & $\begin{array}{c}\text { Load } \\
\text { shedding }\end{array}$ & $\begin{array}{c}\text { Restoration } \\
\text { time }(\mathrm{h})\end{array}$ & $\begin{array}{c}\text { Solution } \\
\text { time }(\mathrm{h})\end{array}$ \\
\hline$[4]$ & 33 bus & Two-stage robust & $21.79 \%$ & 1.40 & 0.187 \\
{$[5]$} & 123 bus & Tabu search & $16.50 \%$ & 0.45 & 1.480 \\
{$[6]$} & 118 bus & Benders decomposition & $18.42 \%$ & 1.00 & 0.152 \\
{$[7]$} & 33 bus & Robust optimization & $29.35 \%$ & 2.15 & 12.463 \\
{$[12]$} & 123 bus & Metaheuristics & $27.66 \%$ & 2.30 & 15.238 \\
{$[19]$} & 33 bus & Two-stage robust & $15.23 \%$ & 0.77 & 2.394 \\
{$[22]$} & 14 bus & Multi-interval & $34.87 \%$ & 2.05 & 10.95 \\
{$[32]$} & 69 bus & Cuckoo search & $21.75 \%$ & 1.10 & 8.51 \\
paper & 33 bus & C\&CG based IO & $\mathbf{1 2 . 8 3 \%}$ & $\mathbf{0 . 3 4}$ & $\mathbf{0 . 0 7 4}$ \\
\hline
\end{tabular}

Table III compares the performance of the model proportion to state-of-the-art. As can be seen, the proposed method has the better performance from computational burden and solution accuracy. To investigate the impact of RCS allocation on the reliability, two sensitivity analyses are done in Fig. 7. As the number of switches installed on the network increases, the system reliability improves accordingly. This confirms that proliferation of RCS can lead to lower load shedding because of reducing the on-fault area and restoration time. This implies that small $\mu \mathrm{Gs}$ are more resistant against the unexpected contingencies.
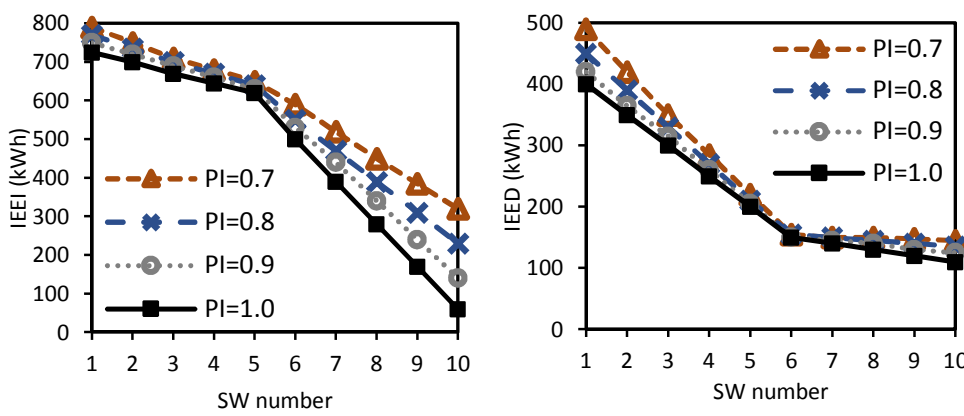

Fig. 7. Sensitivity analysis of switch placement on reliability of $\mu \mathrm{Gs}$.

\section{CONCLUSION AND DISCUSSION}

What was investigated in this paper is the resiliency-oriented planning of distribution networks on the basis of reconfigurable $\mu \mathrm{Gs}$. The main intention of the proposed problem is to divide the network into the small scale zones, taking into account both normal and abnormal operation states of the system. The proposed model provides insight and inspiration regarding how to combine the $\mu \mathrm{Gs}$ planning and its multi-period operation problem at both interconnected and islanded modes. The problem is exercised under uncertain conditions in conjunction with renewable generation via an adjustable interval optimization to create a fair trade-off between solution accuracy and computational burden.

As can be intuitively deduced from evidences, specific features of the proposed model proportion to conventional $\mu \mathrm{Gs}$ planning approaches are listed as below:

$>$ Decision on investment and number of $\mu G s$ : The proposed framework can determine whether the profit obtained from $\mu \mathrm{Gs}$ operation would lead to return on the DER investment cost (i.e., cost-benefit analysis), and would further justify or decline the economic viability of the $\mu \mathrm{Gs}$ deployment in distribution systems. Besides, the proposed model is able to determine the best possible number of $\mu \mathrm{Gs}$ and also tie lines between $\mu \mathrm{Gs}$ for different operation conditions.

$>$ Optimal portfolio and selection of DER units: The model can optimally choose an appropriate combination of various DER portfolio to satisfy economic and reliability considerations of system alongside minimizing the total planning cost and for further enabling a seamless islanding. Increasing the diversity of DERs increases the flexibility and reliability of the $\mu \mathrm{Gs}$ in the face of different short-term operating conditions.

Inclusion of severe uncertainty: Data uncertainty is addressed in the operation stage by assuming that uncertain parameters belong to bounded convex uncertainty sets and minimizing the deviations of the objective function from its expected value. Executing interval optimization protects the $\mu \mathrm{Gs}$ versus risks associated with uncertainties and hedges the $\mu \mathrm{Gs}$ against the risk if compared with the conventional deterministic method.

$>$ Operational flexibility and robustness: The proposed method excerpts different types of generation portfolio to increase the flexibility and controllability of system by overlapping each other's weaknesses. The ESS units, for example, can be regarded as buffers that enhance flexibility in responding to fluctuations pertaining to renewable productions and enhance the resiliency of $\mu \mathrm{G}$ against contingencies.

$>$ Computational tractability and time-scale considerations: For the sake of convexity, the problem is formulated as an exact computationally efficient MILP where the short-term operation and long-term investment problems are decoupled using a novel C\&CG method. The short-term operation includes daily operation of $\mu \mathrm{Gs}$ under electricity market, while the long-term problem incorporates decisions on DER units and remotely controlled switches investments. These two different problems are linked through optimality cuts generated in the operation subproblem.

$\mu G$ islanding consideration: One of the most salient benefits of $\mu \mathrm{G}$ deployment is its islanding capability that can supply the critical demand during emergency conditions, thus this feature is efficiently incorporated into the planning problem. 
In this regard, two novel reliability-oriented indices have been suggested to measure the ability of $\mu$ Gs for supplying its demand over islanding mode. These indices are constrained into the objective of the problem for reliability considerations. The results confirmed that the $\mu \mathrm{Gs}$ by utilizing the local DERs and sectionalizing on-fault area from unscathed zones can quickly restore the power system to pre-event state as soon as possible aimed at escaping from full blackouts.

\section{REFERENCES}

[1] H. Farhangi, "The path of the smart grid," IEEE power and energy magazine, vol. 8, no. 1, pp. 18-28, 2010.

[2] Y. Yoldaş, et al., "Enhancing smart grid with microgrids: Challenges and opportunities," Rene. \& Sustain. Energy Reviews, vol. 72, pp. 205-214, 2017.

[3] IEEE guide for design, operation, and integration of distributed resource island systems with electric power systems, IEEE Std. 1547.4, pp. 1-54, 2011

[4] SA. Arefifar, et al., "DG mix, reactive sources and energy storage units for optimizing microgrid reliability and supply security," IEEE Transactions on Smart Grid, vol. 5, no. 4, pp. 1835-1844, 2014.

[5] Z. Wang, J. Wang, "Self-healing resilient distribution systems based on sectionalization into microgrids," IEEE Transactions on Power Systems, vol. 30, no. 6, pp. 3139-3149, 2015.

[6] A. Khodaei, M. Shahidehpour, "Microgrid-based co-optimization of generation and transmission planning in power systems," IEEE Transactions Power Systems, vol. 28, no. 2, pp. 1582-1590, 2013.

[7] Z. Wang, et al., "Robust optimization based optimal DG placement in Microgrids," IEEE Trans. on Smart Grid, vol. 5, no. 5, pp. 21732182, 2014.

[8] H. Lotfi, A. Khodaei, "AC versus DC microgrid planning," IEEE Transactions on Smart Grid, vol. 8, no. 1, pp. 296-304, 2017.

[9] A. Soroudi, T. Amraee, "Decision making under uncertainty in energy systems: State of the art," Renewable and Sustainable Energy Reviews, vol. 28, pp. 376-384, 2013.

[10] A. Soroudi, M. Ehsan, "IGDT based robust decision making tool for DNOs in load procurement under severe uncertainty," IEEE Transactions on Smart Grid, vol. 4, no. 2, pp. 886-895, 2013.

[11] C. Zhang, Y. Xu, Z. Yang Dong, "Probability-Weighted robust optimization for distributed generation planning in microgrids," IEEE Transactions on Power Systems, vol. 33, no. 6, pp. 7042-7051, 2018.

[12] SA. Arefifar, et al, "Comprehensive operational planning framework for self-healing control actions in smart distribution grids," IEEE Transactions on Power Systems, vol. 28, no. 4, pp. 4192-4200, 2013.

[13] H. Qiu, et al., "Bi-level two-stage robust optimal scheduling for ac/dc hybrid multi-microgrids," IEEE Trans. on Smart Grid, vol. 9, pp. 5455-5466, 2018.

[14] B. Zhao, et al., "Robust optimal dispatch of ac/dc hybrid microgrids considering generation and load uncertainties and energy storage loss," IEEE Transactions on Power Systems, vol. 33, no. 6, pp. 59455957, 2018.

[15] H. Qiu, et al., "Multi-time-scale rolling optimal dispatch for ac/dc hybrid microgrids with day-ahead distributionally robust scheduling," IEEE Transactions on Sustainable Energy, vol. PP, pp. 1-11, 2018.

[16] FS. Gazijahani, S. Najafi Ravadanegh, J. Salehi, "Stochastic multiobjective model for optimal energy exchange optimization of networked microgrids with presence of renewable generation under risk-based strategies," ISA Transactions, vol. 73, pp. 100-111, 2018.
[17] B. Zhang, et al., "Robust optimization for energy transactions in multi-microgrids under uncertainty," Applied Energy, vol. 217, pp. 346-360, 2018.

[18] H. Qiu, et al., "Bi-level two-stage robust optimal scheduling for ac/dc hybrid multi-microgrids," IEEE Transactions on Smart Grid, vol. 9, no. 5, pp. 5455-5466, 2019.

[19] FS. Gazijahani, J. Salehi, "Reliability constrained two-stage optimization of multiple renewable-based microgrids incorporating critical energy peak pricing demand response program using robust optimization approach," Energy, vol. 161, pp. 999-1015, 2019.

[20] A. Khodaei, S. Bahramirad, M. Shahidehpour, "Microgrid planning under uncertainty," IEEE Transactions on Power Systems, vol. 30, no. 5, pp. 2417-2425, 2016.

[21] FS. Gazijahani, J. Salehi, "Robust design of microgrids with reconfigurable topology under severe uncertainty, IEEE Transactions on Sustainable Energy, vol. 9, no. 2, pp. 559-569, 2018.

[22] H. Qiu, et al, "Multi-interval-uncertainty constrained robust dispatch for $\mathrm{AC} / \mathrm{DC}$ hybrid microgrids with dynamic energy storage degradation," Applied Energy, vol. 228, pp. 205-214, 2018.

[23] T. Lv, Q. Ai, Y. Zhao, "A bi-level multi-objective optimal operation of grid-connected microgrids," Electric Power Systems Research, vol. 131, pp. 60-70, 2017.

[24] T. Shekari, S. Golshannavaz, F. Aminifar, "Techno-economic collaboration of PEV fleets in energy management of microgrids," IEEE Transactions on Power Systems, vol. 32, no. 5, pp. 3833-3841, 2017.

[25] P. Salyani, et al., "Chance constrained simultaneous optimization of substations, feeders, renewable and nonrenewable distributed generations in distribution network," Elec. Power Syst. Res., vol. 158, pp. 56-69, 2018.

[26] Transmission and Distribution Committee. IEEE Guide for Electric Power Distribution Reliability Indices. IEEE Std. $1366^{\mathrm{TM}}$ 2012, 2012

[27] S. Wang, Z. Li, L. Wu, M. Shahidehpour, Z. Li, "New metrics for assessing the reliability and economics of microgrids in distribution system," IEEE transactions on power systems, vol. 28, no. 3, pp. 2852-2861, 2013.

[28] R. Moore, R Kearfott, M. Cloud. Introduction to interval analysis. Siam 110, Edition 2, 2009.

[29] A. Bhurjee, et al., "Efficient solution of interval optimization problem," Math. Methods of Operations Research, vol. 76, no. 3, pp. 273-288, 2012.

[30] B. Zeng, Bo, L. Zhao," Solving two-stage robust optimization problems using a column-and-constraint generation method, "Operations Research Letters, vol. 41, no. 5, pp. 457-461, 2013.

[31] The General Algebraic Modeling System (GAMS) Software Website, 2019. [Online]. Available: http://www.gams.com/dd/docs/solvers/ cplex.pdf.

[32] FS. Gazijahani, J. Salehi, "Optimal bilevel model for stochastic risk-based planning of microgrids under uncertainty," IEEE Transactions on Industrial Informatics, vol. 14, no. 7, pp. 3054-3064, 2018.

[33] Electricity Market Today-IESO, 2018. [Online].http://www.ieso.ca/ Power-Data/Price-Overview/Hourly-Ontario-Energy-Price. 